ReBEn, $34 \quad 125-137,1981$

\title{
INTRODUÇÃO À ENFERMAGEM \\ IMPLANTAÇÃO DA METODOLOGIA ASSISTENCIAL DE ENFERMAGEM NO IASERJ
}

\author{
Lódia Barreto de Resende* \\ Véra Rodrigues Oliveira de Andrade** \\ Conceição Enid Imbiriba ***
}

ReBEn/01

RESENDE, L.B. e Colaboradoras - Implantaçāo de Metodologia Assistencial de Enfermagem no IASERJ. Rev. Bras. Enf.; DF, 34 : 123-137, 1981.

\section{I - INTRODUÇÃO}

É evidente que observaçōes científicas sobre a assistência de enfermagem têm-se tornado atualmente objeto de estudo, investigação e aplicação, anunciando novas eras na educação teórico-prática do enfermeiro.

Essas investigaçōes tendem a se aperfeiçoar, para que novas experimentaçōes técnicas e métodos mais funcionais, criativos e adequados possam surgir, numa tentativa de oferecer uma assistência integral em todos os níveis de assistência.

O desafio da década de 80 diz respeito ao aparecimento de novas açōes relacionadas à saúde, e novas perspectivas mundiais já começam a ser di- mensionadas, no sentido da valorização e preservação da existência humana.

A partir do exposto, as autoras elaboraram o presente trabalho, cuja idéia principal é a de oferecer alguns subsídios e recursos que visem aprimorar a assistência, contribuindo assim para a elevação do nível profissional e do bemestar da sociedade.

Desde 1974 a Divisão de Enfermagem do Hospital Central do IASERJ já demonstrava preocupaçōes em introduzir o Processo de Enfermagem e empenhou-se em proporcionar aos enfermeiros lim curso que despertasse interesse pelo novo método, enfatizando a importância da sua aplicabilidade. Vários esforços foram desenvolvidos no sentido de facilitar a introdução do

Tema livre do XXXII CBEn Brasilia D.F. Brasil/1980.

* Enfermeira coordenadora da Equipe de Educação em Serviço do Hospital Central do IASERJ. (Relatora)

** Enfermeira da Equipe de Educação em Serviço e Professora da Faculdade de Enfermagem da UERJ.

*** Enfermeira Chefe de Turma Técnica da Dermatologia e Pneumólogia do Hospital Central do IASERJ. 
RESENDE, L.B. e Colaboradoras - Implantação de Metodologia Assistencial de Enfermagem no IASERJ. Rev. Bras. Enf.; DF, 34 : 123-137, 1981.

Processo de Enfermagem; dentre as diversas providências adotadas, foi selecionado um grupo de funcionários, que recebeu treinamento específico para exercer as tarefas de secretário, ficando assim o enfermeiro com mais liberdade para dar continuidade às atividades assistenciais.

Outro valor considerado para a implantação do novo processo de assistência é a possibilidade que o método oferece de avaliaçōes constantes, as quais, por sua vez, servem como base de aperfeiçoamento no cuidado prestado ao paciente.

Ciente da importância desse novo método de assistência, a Divisão de Enfermagem do Hospital Central do IASERJ resolveu traçar diretrizes gerais e metas relativas à implantação do Processo de Enfermagem, com bases na metodologia científica.

Para que os objetivos pudessem ser alcançados, foi elaborado um questionário dirigido aos enfermeiros que situasse dentro da possível problemática existente a implantação do novo método assistencial de enfermagem.

\section{II - REVISAXO DE LITERATURA}

Horta (6) conceitua a Enfermagem como a ciência e a arte de assistir o ser humano no atendimento de suas necessidades básicas; de torná-lo independente desta assistência através da educação; recuperar, manter e promover sua saúde pela colaboração cor: outros grupos profissionais.

Apresenta ainda as seguintes etapas do Processo de Enfermagem:

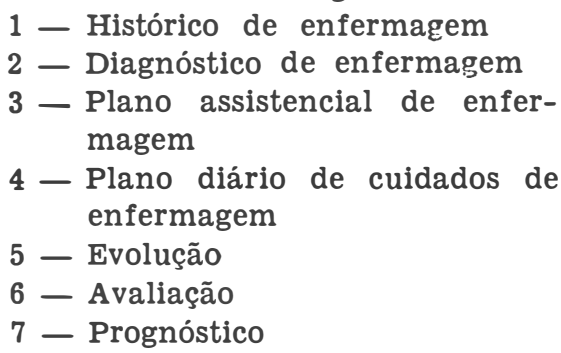

Ainda visualizando a enfermagem dentro de uma perspectiva sistêmica, R. Paim (21) assim a define: "Como a ciência e a arte que trata da identificação dos problemas que lhe são afetos, consoante critério da Organização Mundial de Saúde; realiza ou co. ordena açōes no sentido de solucionálos, atuando simultaneamente sobre o sistema Homem."

Apresenta o processo cibernético de enfermagem cujas etapas foram assim designadas:

1 - Histórico de enfermagem

2 - Evolução de enfermagem

3 - Prognóstico de enfermagem

4 - Prescrição de enfermagem

5 - Plano assistencial global de enfermagem

6 - Plano diário de cuidados de enfermagem

7 - Plano de alta terapêutica de enfermagem

8 - Avaliação de enfermagem

9 - Retroação (feed back)

Paim, L. (18) sugere uma classificação de prescriçōes de enfermagem atendendo a três elementos:

- Dominio

- Propósito

- Dependência

Ao domínio equivale a ênfase da prescrição em termos de necessidade estas atendidas como psico-biológicas, psico-sociais e psico-espirituais.

Quanto ao propósito, refere-os como:

1 - Preservação do equilíbrio

2 - Prevenção do desequilíbrio

3 - Detecção de sinais e sintomas

4 - Promoção de equilíbrio

5 - Reintegração do equilíbrio

6 - Propósito relativo a implantação de prescrição médica.

Finalmente, quanto a dependência, utilizou a classificação de Horta, ou seja, em cinco níveis:

$$
\begin{aligned}
& 1 \text { - Total } \\
& 2 \text { - Ajuda } \\
& 3 \text { - Orientação }
\end{aligned}
$$


RESENDE, L.B. e Colaboradoras - Implantaçāo de Metodologia Assistencial de Enfermagem no IASERJ. Rev. Bras. Enf.; DF, 34 : 123-137, 1981.

4 - Encaminhamento

5 - Supervisão

Daniel, L. (3) conceitua:

1 - Histórico, como "o levantamento sistemático de informaçōes do paciente, familia e comunidade e observaçōes adquiridas através do exame físico do paciente".

2 - Diagnóstico, como sendo "a determinação das necessidades básicas afetadas".

3 - Plano terapêutico, como "o estudo prévio e teórico-prático de problemas inerentes a atividades especificas que se querem desenvolver no atendimento total e diário de enfermagem ao paciente".

4 - Evolução, como "o estudo ou consideração contínua e progressiva das respostas ou reaçōes do paciente, família e comunidade à assistência de enfermagem prestada".

5 - Avaliação, como "um levantamento ou verificação dos procedimentos de enfermagem utilizados e dos resultados obtidos no atendimento das necessidades básicas da pessoa humana".

6 - Prognóstico, como "uma estimativa da capacidade, perspectiva e probabilidades do paciente, cliente, e família, de atender às suas próprias necessidades básicas, após a implantação do plano terapêutico de enfermagem".

Lambertsen (11) conceitua a enfermagem como: "um processo dinâmico, terapêutico e educativo em satisfazer às necessidades de saúde da sociedade. Sua função distinta refere-se às respostas fisiológicas e psico-sociais à saúde que podem ou fazem resultar em um estado de dependência e outro para satisfazer necessidades que estão normalmente dentro do potencial do indivíduo ou família. Em um relacionamento terapêutico e educativo o enfermeiro assiste o individuo e/ou familia em alcançar seu potencial de auto direção da saúde".
Ainda posicionando a Enfermagem, define-a como "uma ciência que leva ao ser humano o conhecimento necessário para a preservação da saúde e da família, assegurando assim a perpetuação da espécie".

Little e Carnevalle (13) vêem o Processo de Enfermagem como passos seguidos no planejamento do cuidado ao paciente, sendo um processo de identificação de problemas.

Lewis (12) afirma que o Processo de Enfermagem é base para o planejamento do cuidado do paciente e vê isso como processo de solução de problemas, envolvendo uma apreciação sistemática do paciente para o cuidado de enfermagem.

Pahaneuf (22) define o Processo de Enfermagem como tomada de medidas mais ou menos importantes na direção do cuidado do paciente.

Segundo Hargreeves, (4) o Processo de Enfermagem é aplicável em todas as situaçōes de enfermagem. As Ciências e habilidades fundamentais devem ser consideradas no Processo de Enfermagem. A individualização do cuidado é um dos critérios pelos quais podemos julgar qualidades do cuidado de enfermagem prestados aos nossos pacientes.

Yura (27) trata o Processo de Enfermagem como uma forma sistemática de determinar os problemas a fim de elaborar planos para resolvê-los, implementar o mesmo e avaliar a extensão do efeito desses planos na resolução dos problemas identificados.

Poole (23) critica os enfermeiros que na sua maioria responde às solicitacōes com a frase "NĀO TENHO TEMPO". Conclui em seu trabalho que a maioria desses profissionais dizem não ter tempo porque estão entregues ao cumprimento de rotinas hospitalares.

Arzani (1) trata sobre cálculo de pessoal de enfermagem e descreve a necessidade de ser utilizado um método efetivo e prático para este cálculo. $\mathrm{Su}$ - 
RESENDE, L.B. e Colaboradoras - Implantaçāo de Metodologia Assistencial de Enfermagem no IASERJ. Rev. Bras. Enf.; DF, $34: 123-137,1981$.

gere que, para qualquer cálculo dessa natureza, deve-se emprestar fundamental importância à quantidade e à situação dos pacientes.

Mc Cain (16) critica a tendência çue os enfermeiros têm de utilizar somente a intuição. Compara a enfermagem às demais profissões e conclui que somente os enfermeiros ainda não definiram seus métodos na prática profissional.

De acordo com Cianciarullo (2), "a complexidade e a diversificação crescente das teorias de enfermagem dificultam uma abordagem dirigida essencialmente para o Histórico de Enfermagem. A elaboração de um instrumen. to para a coleta sistemática destes dados enquadra-se ainda na necessidade de uma metodologia de trabalho para I!ma atuação efetiva e coerente da enfermeira".

Orlando (17) vê o Processo de Enfermagem como baseado na interação enfermeiro-paciente. O Processo bastiase no diagnóstico das necessidades do jaciente e dos fatores que as influênciam. Ele é decidido depois que o Enfermeiro conhece o significado do comportamento do paciente e reconheceu as atividades específicas que são requeridas para satisfazer suas necessidades.

\section{ITI - METODOLOGIA}

A presente pesquisa foi realizada no Hospital Central do Instituto de Assistência dos Servidores do Estado do Rio de Janeiro, no período de julho a setembro de 1979, visando identificar, analisar e interpretar as dificuldades encontradas na implantação da Metodologia Científica.

O referido Hospital dispōe de qua. trocentos e setenta e sete leitos, distribuídos entre o Pavilhão Clínico, $\mathrm{Pa}$ vilhão Cirúrgico e Emergência, sendo a seguinte a distribuição de pessoal por categoria funcional: 150 Enfermeiros,
5 Obstetrizes, 374 Auxiliares de Enfermagem e 155 Atendentes.

Como etapa inicial, realizou-se uma pesquisa de campo, cujo instrumento, utilizado para a coleta de dados, foi um questionário contendo 18 perguntas fechadas e 2 perguntas abertas (anexo 1), como objetivo de verificar quais as variáveis que interferiam no processo de implantação da Metodologia Assistencial.

Dos cento e cinqüenta enfermeiros lotados na instituição, setenta e quatro responderam ao questionário.

\section{IV - INTERPRETAÇÃO DOS RESULTADOS}

Após a pesquisa realizada, foi constatado que das setenta e quatro enfermeiras, $69 \%$ receberam aulas sobre 0 Processo de Enfermagem durante seu curso de graduação e $31 \%$ não receberam (tab. I). Sendo que das que receberam aulas, $94 \%$ tiveram a oportunidade de elaborá-lo e $6 \%$ não tiveram (tab. II)

Em relação à participação do estudo dirigido sobre a Metodologia Assistencial, realizada na instituição, $39 \%$ não participaram e $4 \%$ deixaram em branco (tab. III). Nos cursos realizados em outras instituições, $3 \%$ fizeram e $97 \%$ não fizeram (tab. IV).

Os cursos citados foram: Metodologia Assistencial do Centro de Ciências da Saúde, da U.E.R.J. do Congresso de Enfermagem - BELEM - (tab. V).

Sobre as vantagens que o processo traz para a profissão, 9\% dos enfermeiros responderam que delimita a área de ação do enfermeiro, $31 \%$ proporciona maior autonomia profissional, $59 \%$ dá um cunho científico ao trabalho de equipe, $0 \%$ não traz vantagens e $1 \%$ deixou em branco (tab. VI).

No que diz respeito à segurança pessoal na aplicação do processo, os enfermeiros posicionaram-se da seguinte maneira: $38 \%$ seguro, $4 \%$ inse- 
RESENDE, L.B. e Colaboradoras - Implantação de Metodologia Assistencial de Enfermagem no IASERJ. Rev. Bras. Enf.; DF, 34 : 123-137, 1981.

guro, $58 \%$ com alguma dúvida (tab. VII).

Quanto à realização do Processo de Enfermagem nas clínicas, vemos que: $80 \%$ realizam e $20 \%$ não realizam (tab. VIII)

Sobre qual o critério adotado, verificamos que: $\mathbf{3 8 \%}$ dos enfermeiros aplicam o processo em todos os pacienentes, $18 \%$ só nos pacientes graves, $24 \%$ a título de experiência e $20 \%$ não responderam (tab. IX).

No que tange à vantagem que o Processo de Enfermagem trouxe para o serviço, $23 \%$ acham que facilita a determinação do tipo de Assistência de Enfermagem prestada, 9\% acham que não houve vantagem e $13 \%$ não responderam (tab. X).

Em relação à assistência recebida pelo paciente com o processo $42 \%$ dos enfermeiros acham que melhora bastante o nivel, $35 \%$ melhora razoavelmente, $8 \%$ não altera o nível e $15 \%$ não responderam (tab. XI).

Sobre os melhores recursos deverão ser utilizados no treinamento de enfermeiros, ficaram assim colocados: $41 \%$ para cursos, $10 \%$ para palestras, $9 \%$ para seminários, $40 \%$ para reuniōes científicas com estudo de caso (tab. XII).

Relacionando as fases do processo e o nivel de dificuldade, verificamos que: $12 \%$ dos enfermeiros acham que é o histórico, $32 \%$ o diagnóstico, $25 \%$ o plano assistencial, $4 \%$ o plano de cuidado diário, $5 \%$ a evolução e $22 \%$ não encontram dificuldade (tab. XIII).

Quanto ao nivel de conhecimento enfermeiro-paciente que o Histórico possibilita; $12 \%$ responderam superficial, $83 \%$ profundo, e $5 \%$ não responderam (tab. XIV).

Com referência à maior dificuldade na elaboração do diagnóstico de enfermeiro; $14 \%$ responderam estar na identificação dos problemas, $28 \%$ na determinação das necessidades básicas, $25 \%$ na determinação do grau de dependência, $28 \%$ não encontraram difi- culdades e 5\% não responderam (tab. $\mathrm{XV})$.

Com relação à dificuldade na elaboração do plano assistencial, $25 \%$ responderam que está na curta permanência do paciente no hospital, $59 \%$ no pouco tempo disponivel para a elaboração do mesmo, 8\% não encontram dificuldades, $6 \%$ outras dificuldades não expostas e $2 \%$ não responderam (tab. XVI) .

Quanto à dificuldade na elaboração do plano de cuidado diário: $14 \%$ alegam a visita médica, $18 \%$ falta de planejamento no horário da visita médica, $43 \%$ não encontraram dificuldades, $15 \%$ outras dificuldades não expostas e $10 \%$ não responderam (tab. XVII).

No entendimento dos enfermeiros, quanto à dificuldade na elaboração da evolução: $50 \%$ responderam estar nas deficiências das anotaçōes de enfermagem, $18 \%$ falhas na elaboração do plano de cuidado diário, $23 \%$ não encontraram dificuldade, $4 \%$ outras dificuldades não expostas e $5 \%$ não responderam (tab. XVIII).

Em relação às barreiras existentes para a implantação total do Processo de Enfermagem, 49\% não identificaram barreiras e $51 \%$ mencionaram a seguir as seguintes barreiras: planta física deficiente $20 \%$, falta de profissionais $10 \%$, escassez de tempo $10 \%$, deficiência nos registros de enfermagem $30 \%$ e pessoal auxiliar não treinado $30 \%$ (tab. XIX).

Sendo estas as sugestōes colocadas para suplantar estas barreiras: conscientização e supervisão das Chefes de Turma Técnica $50 \%$, elevação do número de funcionários habilitados $20 \%$ e reciclagem da equipe $30 \%$ (tab. $\mathrm{XX}$ ).

\section{$\mathrm{V}-$ CONCLUSOES}

Ao final do trabalho, as autoras concluiram que, apesar das inúmeras bärreiras encontradas na implantação da metodologia assistencial, estas não constituiram obstáculo capazes de im- 
RESENDE, L.B. e Colaboradoras - Implantação de Metodologia Assistencial de Enfermagem no IASERJ. Rev. Bras. Enf.; DF, 34 : 123-137, 1981.

pedir a aceitação e o reconhecimento do novo método.

Um dos fatores relevantes que ajudaram a superar estas barreiras, foi a conscientização por parte dos enfermeiros quanto à importância do planejamento da assistência de enfermagem, que por certo conduzirá o paciente o quanto antes a reassumir o seu papel junto à família e à sociedade.

\section{VI - RECOMENDAÇŐES}

Que as instituiçōes hospitalares apóiem as experimentaçōes científicas de enfermagem com a finalidade de implantar a nova Metodologia assistencial, visando oferecer ao paciente um nível cada vez mais elevado de assistência.

Que todo enfermeiro procure valorizar, compreender e aplicar o processo de enfermagem, a fim de proporcionar ao paciente uma assistência centrada nas necessidades básicas humanas.

Que os enfermeiros promovam efetiva interação entre a equipe de saúde, a fim de conjugar esforços no sentido de assegurar ao paciente uma assistência integral visando também a um verdadeiro reconhecimento profissional.

Que as Chefias de serviço estejam empenhadas em promover situaçōes que visem assegurar a continuidade da assistência planejada.

\section{B I B L I O G R A F I A}

1. ARZANI, M. E. EI cálculo de Personal de enfermeria en la problematica de la atencion medica. Revista Argentina de enfermeria. 3(4), out., 1976.

2. Cianciarullo, T. I. O Histórico de enfermagem no contexto do processo de enfermagem. Enfermagem em Novas Dimensōes. 2(4), 187-189, 1976.

3. DANIEL, L. F. A Enfermagem Planejada. São Paulo, 1977.

4. HARGREeVES, I. O Processo de Entermagem: A chave para o cuidado individualizado - Nursing Times August. XXVIII, 89-91, 1975.

5. HOHANA, J. O mundo e eu. 6. ed. Rio de Janeiro, Agir, 1976.

6. HORTA, W. A. Contribuição a uma teoria de Enfermagem. Revista Brasileira de Enfermagem. XXIII. 3(6), jul./dez., 1970.

7. ........ O Processo de Enfermagem. Fundamentação e Aplicação. Enfermagem em Novas Dimensōes. 1(1) $16,1975$.

8. ......... O Processo de Enfermagem Representação gráfica. Enfermagem em Novas Dimensōes. 3(2), 75-77, 1977. 9....$\ldots \ldots$ A Metodologia do Processo de Enfermagem. Revista Brasileira de Enfermagem. XXXIV (6), 81-95 out./dez., 1971.

10. KRON, T. Manual de Enfermagem. Nueva Editorial Interamericana México, 1973

11. LAMBERTSEN, E. C. Nursing Team Organization and Functioning. Teacher College, New York, 1953.

12. LEWIS. L. Planning patient care W.M.C. Brow Company, Publishers, Dubuque, Iowa, Loma Linda University, 1970.

13. Lithe, D. E. \& CARNEVALLE. D. L Nursing Care Planning. 2. ed. Philadelphia, Lippincott Company, 1972

14. MARTINS, C.B.G. Avaliação da Assistência. Enfermagem em Novas Dimensōes. 1(3), 113-118, 1975.

15. MASLOW, A. H. Introdução à Psicologia do Ser. 2. ed. Eldorado, Rio de Janeiro.

16. MC CAIN, F. R. Nursing by assessment not intuiton. American Journal of Nursing. 65(4), 82-85, 1965.

17. ORLANDO, I. J. The Dinamic Nurse. Patient Relations. New York, 2 ed. 1972. 
RESENDE, L.B. e Colaboradoras - Implantação de Metodologia Assistencial de Enfermagem no IASERJ. Rev. Bras. Enf.; DF, 34 : 123-137, 1981.

18. PAIM, L. et alii. Iniciamento à Metodologia do Processo de Enfermage:n. Rio de Janeiro, ABEn, 1974.

19. PAIM, L. A Prescrição de Enfermagem. Unidade valorativa do plano de cuidados. Dissertação de Mestradio apresentada à Escola Ana Nery da UFRJ, 1975.

20. PAIM, L. Plano Assistencial e Prescriçōes de Enfermagem. Revista Brasileira de Enfermagem. 29, 66-82, Brasília, 1976.

21. PAIM, R. Problemas de Enfermagem e Terapia Centrada nas necessidades do paciente. Rio de Janeiro, Uniảo dos Cursos Cariocas, 1979.

22. PAHANEUF, M. C. A Nursing cuid. + Method., Nursing Outtosk, 12, may, 1964.
23. POOLE, E. P. "Enfermeiro, prove-me de quem você cuida". The canadian Nurse. Fev., 1970.

24. RIBEIRO, C. V. Organização do Serviço de Enfermagem. Revista Brasileira de Enfermagem. 26(3), 121 abr./Jun., 1973.

25. RUIZ, J. A. Metodologia Cientifica: guia para eficiência nos estudos. São Paulo, Atlas, 1978

26. SALOMON, D. V. Como fazer uma monografia. Minas Gerais, Instituto de Psicologia da Universidade Católica, 1971.

27. YURA, H.W.M.B. The Nursing Process. 2. ${ }^{\text {a }}$ ed. New York. Appleton Century, Crofts, 1973.

\section{$A N E \times O \quad$ \\ HOSPITAL CENTRAL DO IASERJ}

DIVISĀO DE ENFERMAGEM - TREINAMENTO EM SERVIÇO

\section{QUESTIONARIO SOBRE A IMPLANTAÇÃO DO PROCESSO DE ENFERMAGEM}

$$
\text { POPULAÇAO - ENFERMEIROS }
$$

1 - Durante o seu curso de graduação, teve aulas sobre o Processo de Enfermagem?
Sim
Não

2 - Em caso afirmativo, teve oportunidade de elaborá-lo?

$\operatorname{Sim}$

Não

3 - Participou do estudo dirigido, realizado na instituição, sobre a Metodologia Assistencial?
Sim
Não

4 - Fez algum curso sobre o assunto?

$\operatorname{Sim}$

Não

5 - Em caso afirmativo, cite o mesmo 
RESENDE, L.B. e Colaboradoras - Implantação de Metodologia Assistencial de Enfermagem no IASERJ. Rev. Bras. Enf.; DF, $34: 123-137,1981$.

6 - No seu entendimento, quais as vantagens que o processo traz para a sua profissão?

( ) Delimita a área de açāo do enfermeiro

( ) Proporciona uma maior autonomia profissional

( ) Dá um cunho científico ao trabalho da equipe

( ) Não traz vantagens

7 - Em relação à segurança pessoal, qual sua posição quanto à aplicação do processo?

(. ) Segura

( ) Insegura

( ) Com algumas dúvidas

8 - Na sua clínica, o processo de enfermagem vem sendo realizado?
$\operatorname{Sim}$
Não

9 - Qual o critério adotado?

( ) Todos os pacientes

( ) Só os graves

( ) A titulo de experiência

10 - Qual a vantagem que o processo trouxe para o Serviço?

( ) Facilitar a determinação do tipo de assistência de enfermagem a cada paciente

( ) Proporcionar melhor visão e controle da assistência prestado

( ) Não houve

11 - Em relação à assistência recebida pelo paciente com o processo?

( ) Melhora bastante o nivel

( ) Melhora razoavelmente

( ) Não altera o nível

12 - Na sua opinião, quais os recursos que devem ser usados para o treinamento dos enfermeiros?

( ) Cursos

( ) Palestras

( ) Seminários

( ) Reuniōes Científicas com Estudo de Caso

( ) Outros

13 - No seu entendimento, qual a fase do processo de enfermagem mais difícil de ser elaborada?

Histórico

Diagnóstico 
RESENDE, L.B. e Colaboradoras - Implantaçāo de Metodologia Assistencial de Enfermagem no IASERJ. Rev. Bras. Enf.; DF, 34 : 123-137, 1981.
( ) Plano assistencial
( ) Plano de cuidado diário
( ) Evolução
( ) Nenhum

14 - O histórico possibilita um conhecimento, enfermeiro-paciente, em que nivel?
( ) Superficial
( ) Profundo
( ) Nenhum

15 - Qual a maior dificuldade na elaboração do diagnóstico de enf.?
( ) Identificação dos problemas
( ) Determinação das necessidades básicas
( ) Determinação do grau de dependência
( ) Nenhum

16 - Qual a maior dificuldade na elaboração do plano assistencial?

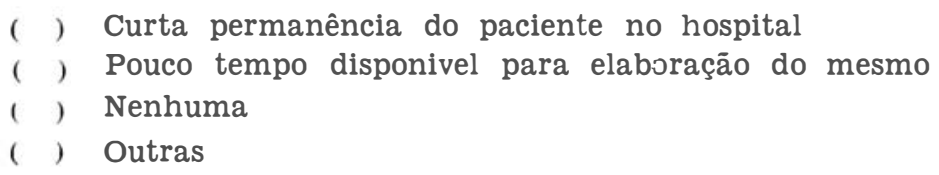

17 - Qual a maior dificuldade na elaboração do plano de cuidado diário?
( ) Dificuldade quanto a visita diária
( ) Falhas na elaboração do plano de cuidado diário
( ) Nenhuma
( ) Outras

18 - Qual a maior dificuldade na elaboração da evolução?
(1) Deficiência das anotaçōes de enfermagem
( ) Falhas na elaboração do plano de cuidados diário.
( ) Nenhuma
() Outras

19 - Quais as barreiras ainda existentes para a implantação total no processo de enfermagem?

20 - Quais as suas sugestōes para suplantar estas barreiras? 
RESENDE, L.E. e Colaboradoras - Implantaçāo da Metodologia Assistencial de Enfermagem no IASERJ. Rev. Bras. Enf.; DF, 34 : 123-137, 1981.

TABELA I

Enfermeiros que durante o curso de graduação receberam aulas sobre o Processo de Enfermagem.

IASERJ - RJ - 1979

\begin{tabular}{cccr}
\hline RECEBERAM & FULAS & $\%$ \\
\hline Sim & 51 & 69 \\
Não & 23 & 31 \\
Total & 74 & 100 \\
& & \\
\hline
\end{tabular}

TABELA II

Enfermeiros que tiveram oportunidade de elaborar o processo de enfermagem durante o curso de graduação.

IASERJ - RJ - 1979

\begin{tabular}{crr}
\hline ELABORAÇAOO DO PROCESSO & F & $\%$ \\
\hline Sim & 48 & 94 \\
Não & 3 & 6 \\
Total & 51 & 100 \\
\hline
\end{tabular}

TABELA III

Enfermeiros que participaram do estudo dírigido, realizado na instituiçāo sobre a Metodologia Científica.

IASERJ - RJ - 1979

\begin{tabular}{lrr}
\hline PARTICIPAÇAO DO ESTUDO DIRIGIDO & F & $\%$ \\
\hline Sim & 42 & 57 \\
Não & 29 & 39 \\
Não respondida & 4 & 4 \\
Total & 88 & 100 \\
\hline
\end{tabular}

TABELA IV

Enfermeiros que participaram de cursos sobre a Metodologia Científica, fora da instituição.

IASERJ - RJ - 1979

\begin{tabular}{ccc}
\hline PARTICIPAÇAOO DE CURSOS & F & $\%$ \\
\hline SIm & & \multicolumn{1}{c}{$\%$} \\
Não & $\mathbf{5 5}$ & $\mathbf{7 5}$ \\
Total & 74 & 100 \\
\hline
\end{tabular}


RESENDE, L.B. e Colaboradoras - Implantaçāo de Metodologia Assistencial de Enfermagem no IASERJ. Rev. Bras. Enf.; DF, $34: 123-137,1981$.

TABELA V

Cursos realizados fora da instituição que contaram com a participação dos enfermeiros.

$$
\text { IASERJ - RJ - } 1979
$$

\begin{tabular}{lcc}
\hline \multicolumn{1}{c}{ C UR S O S } & F & $\%$ \\
\hline Centro de Ciëncias da Saúde & 1 & 1 \\
UFRJ & 1 & 1 \\
Congresso de Enf. (Belém) & 1 & 1 \\
Não respondida & 53 & 97 \\
Total & 74 & 100 \\
\hline
\end{tabular}

TABELA VI

Vantagem que o processo traz para a profissão. IASERJ — RJ — 1979

\begin{tabular}{lrr}
\hline \multicolumn{1}{c}{ V A N T A G E N S } & F & $\%$ \\
\hline Delimita a área de ação do enfermeiro & 9 & 9 \\
Proporciona maior autonomia profissional & 31 & 31 \\
Dá um cunho científico ao trabalho de equipe & 56 & 59 \\
Não traz vantagens & 0 & 0 \\
Não respondido & 1 & 1 \\
Total & 97 & 100 \\
\hline
\end{tabular}

TABELA VII

Posição do enfermeiro em relação à segurança pessoal, quanto à aplicação do processo.

$$
\text { IASERJ - RJ - } 1979
$$

\begin{tabular}{lrr}
\hline \multicolumn{1}{c}{ P O S I Ç A O } & F & $\%$ \\
\hline Segura & 28 & 38 \\
Insegura & 3 & 4 \\
Com algumas dúvidas & 43 & 58 \\
Total & 74 & 100 \\
\hline
\end{tabular}

TABELA VIII

Implantação do Processo de Enfermagem nas clínicas. IASERJ - RJ - 1979

\begin{tabular}{ccc}
\hline I M P L A N T A Ç A O & F & $\%$ \\
\hline Sim & 59 & 80 \\
Não & 19 & 20 \\
Total & 78 & 100 \\
\hline
\end{tabular}


RESENDE, LB. e Colaboradoras - Implantação de Metodologia Assistencial de Enfermagem no IASERJ. Rev. Bras. Enf.; DF, $34: 123-137,1981$.

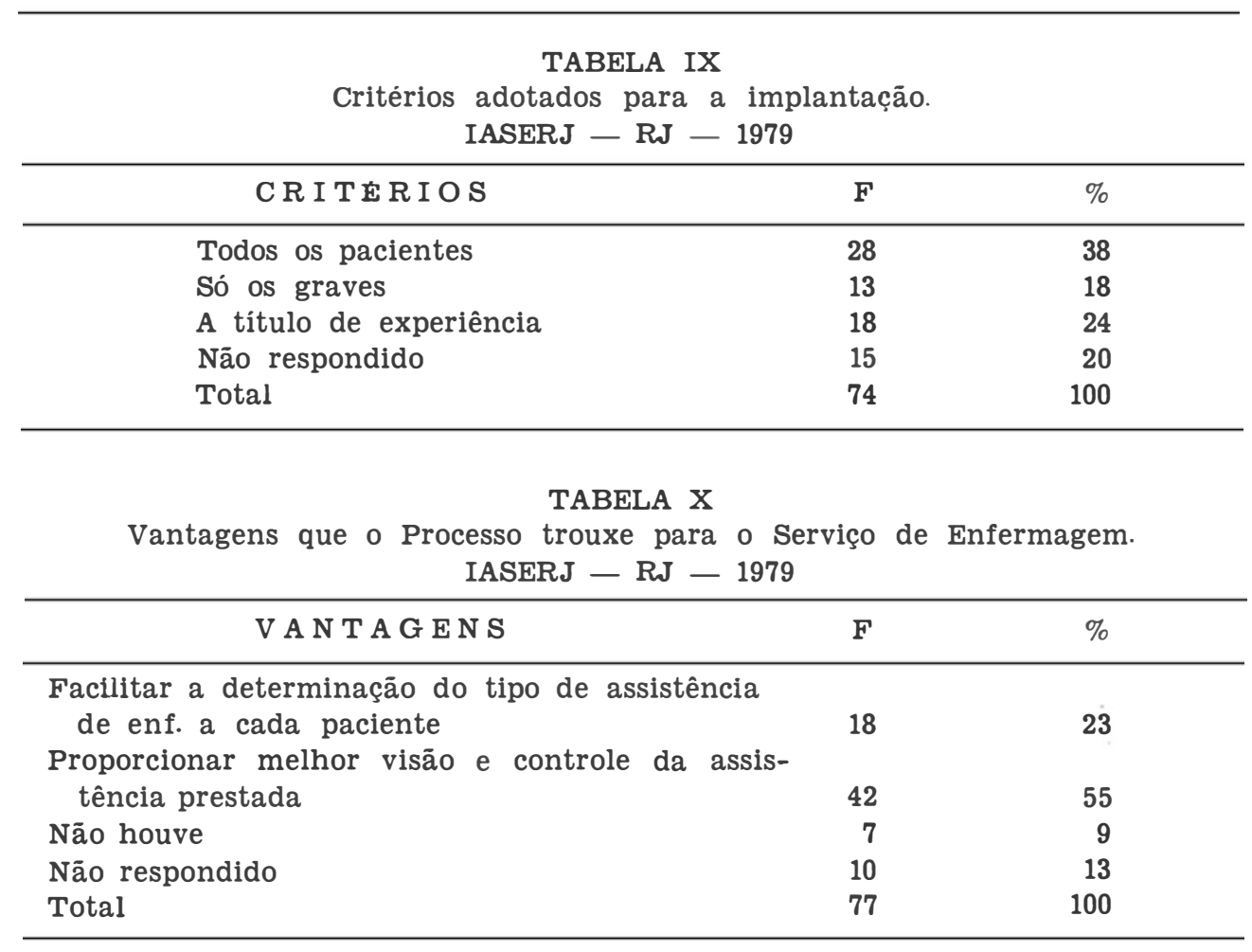

TABELA XI

Vantagens que o processo trouxe para a assistência recebida pelo paciente. IASERJ - RJ - 1979

\begin{tabular}{lrr}
\hline \multicolumn{1}{c}{ V A N T A G E N S } & F & $\%$ \\
\hline Melhora bastante o nivel & 31 & 42 \\
Melhora razoavelmente & 26 & 35 \\
Não altera o nivel & 6 & 8 \\
Não respondido & 11 & 15 \\
Total & 74 & 100 \\
\hline
\end{tabular}

TABELA XII

Recursos que devem ser utilizados para o treinamento de enfermeiros. IASERJ - RJ - 1979

\begin{tabular}{lrr}
\hline RECURSOS PARA TREINAMENTO & F & $\%$ \\
\hline Cursos & 43 & 41 \\
Palestras & 11 & 10 \\
Seminários & 10 & 9 \\
Reuniōes cientificas c/Estudo de caso & 41 & 40 \\
Outros & 0 & 0 \\
Total & 105 & 100 \\
\hline
\end{tabular}


RESENDE, L.B. e Colaboradoras - Implantação de Metodologia Assistencial de Enfermagem no IASERJ. Rev. Bras. Enf.; DF, 34 : 123-137, 1981.

TABELA XIII

Fase do Processo de Enfermagem mais difícil de ser elaborado. IASERJ - RJ - 1979

\begin{tabular}{lrr}
\hline FASES DO PROCESSO & F & $\%$ \\
\hline Histórico & 10 & 12 \\
Diagnóstico & 27 & 32 \\
Plano Assistencial & 22 & 25 \\
Plano de cuidado diário & $B$ & 4 \\
Evolução & 4 & 5 \\
Nenhum & 19 & 22 \\
Total & 85 & 100 \\
\hline
\end{tabular}

TABELA XIV

Nível de conhecimento enfermeiro-paciente que o histórico possibilita. IASERJ - RJ - 1979

\begin{tabular}{lrr}
\hline N I V E L & F & $\%$ \\
\hline Superficial & 9 & 12 \\
Profundo & 61 & 83 \\
Nenhum & 0 & 0 \\
Não respondido & 4 & 5 \\
Total & 74 & 100 \\
\hline
\end{tabular}

TABELA XV

Dificuldades encontradas na elaboração do diagnóstico de enfermagem. IASERJ — RJ - 1979

\begin{tabular}{lrr}
\hline \multicolumn{1}{c}{ D I F I C U L D A D E S } & F & $\%$ \\
\hline Identificação dos problemas & 11 & 14 \\
Determinação das necessidades básicas & 22 & 28 \\
Determinação do grau de dependência & 20 & 25 \\
Nenhum & 22 & 28 \\
Não respondilo & 4 & 5 \\
Total & 79 & 100 \\
\hline
\end{tabular}

TABELA XVI

Dificuldades encontradas na elaboração do plano assistencial. IASERJ - RJ - 1979

\begin{tabular}{lrr}
\hline \multicolumn{1}{c}{ D I F I C U L D A D E S } & F & $\%$ \\
\hline Outra permanência do paciente no hospital & 19 & 25 \\
Pouco tempo disponível para elaborá-lo & 45 & 59 \\
Nenhuma & 6 & 8 \\
Outras & 0 & 0 \\
Não respondido & 7 & 8 \\
Total & 77 & 100 \\
\hline
\end{tabular}


RESENDE, L.B. e Colaboradoras - Implantação de Metodologia Assistencial de Enfermagem no IASERJ. Rev. Bras. Enf.; DF, 34 : 123-137, 1981.

\section{TABELA XVII}

Dificuldades encontradas na elaboração do plano de cuidado diário.

$$
\text { IASERJ - RJ - } 1979
$$

\begin{tabular}{|c|c|c|}
\hline D IF I C U L D A E S & $\mathbf{F}$ & $\%$ \\
\hline Dificuldade quanto à visita diária & 11 & 14 \\
\hline $\begin{array}{l}\text { Falta de planejamento no horário da visita } \\
\text { médica }\end{array}$ & 14 & 18 \\
\hline Nenhuma & 33 & 43 \\
\hline Outras & 12 & 15 \\
\hline Não respondido & 8 & 10 \\
\hline Total & 78 & 100 \\
\hline
\end{tabular}

TABELA XVIII

Dificuldades encontradas na elaboração da Evolução de enfermagem. IASERJ - RJ - 1979

\begin{tabular}{|c|c|c|}
\hline D I F I C U L D D E S & $\mathbf{F}$ & $\%$ \\
\hline Deficiência das anotaçōes de enfermagem & 37 & 50 \\
\hline $\begin{array}{l}\text { Falhas na elaboração do plano de cuidados } \\
\text { diários }\end{array}$ & 13 & 18 \\
\hline Nenhuma & 17 & 23 \\
\hline Outras & 3 & 4 \\
\hline Não respondido & 4 & 5 \\
\hline Total & 74 & 100 \\
\hline
\end{tabular}

\section{TABELA XIX}

Barreiras encontradas para a implantação total do processo de enfermagem.

$$
\text { IASERJ - RJ - } 1979
$$

\begin{tabular}{lrc}
\hline \multicolumn{1}{c}{ BARREIRAS } & F & $\%$ \\
\hline & & \\
Planta física deficiente & 10 & 20 \\
Falta de profissionais & 5 & 10 \\
Escassez do tempo & 5 & 10 \\
Deficiência nos registros de enfermagem & 15 & 30 \\
Pessoal auxiliar nāo treinado & 15 & 30 \\
Total & 50 & 100 \\
\hline
\end{tabular}


RESENDE, L.B. e Colaboradoras - Implantação de Metodologia Assistencial de Enfermagem no IASERJ. Rev. Bras. Enf.; DF, $34: 123-137,1981$.

TABELA XX

Sugestões apresentadas para suplantar as barreiras.

IASERJ - RJ - 1979

\begin{tabular}{|c|c|c|}
\hline S U G E T T O E S & $\mathbf{F}$ & $\%$ \\
\hline $\begin{array}{l}\text { Conscientização e Supervisão das Chefes de } \\
\text { turma técnica }\end{array}$ & 25 & 50 \\
\hline Elevação do número de funcionários habilitados & 10 & 20 \\
\hline Reciclagem da equipe & 15 & 30 \\
\hline Total & 50 & 100 \\
\hline
\end{tabular}

